\title{
ANÁLISE DO PROCESSO DE PRODUÇÃO DE METANOL ATRAVÉS DAS CLÁSSICAS EQUAÇÕES DE ESTADO TERMODINÂMICAS
}

\author{
C. G. A. MISAEL ${ }^{1}$, J.S.B.SANTOS ${ }^{1}$, C. B. CAVALCANTI ${ }^{1}$, H. BISPO ${ }^{1}$ \\ ${ }^{1}$ Universidade Federal de Campina Grande, Unidade Acadêmica de Engenharia Química \\ E-mail para contato: carla.misae194@gmail.com
}

\begin{abstract}
RESUMO - A modelagem termodinâmica de processos permite, através de equações de estado, a previsão do comportamento de propriedades intensivas, tais como entalpia, entropia, volume molar, dentre outras propriedades correlatas. Os resultados obtidos são de grande importância para a descrição dos mais variados processos, tendo em vista que a maioria das substâncias não possuem suas propriedades tabeladas. Dessa forma, com o objetivo de verificar com a máxima exatidão possível o comportamento dos gases reais, a modelagem computacional de uma mistura proveniente de um reator para produção de metanol simulado em plataforma Aspen Plus, será estuda através da clássica equação Peng-Robinson. Os resultados demonstram que as equações de estados apresentam bons resultados para previsão das propriedades intensivas do processo, quando comparadas a equação de Peng-Robison, a qual possui seu melhor desempenho quando a temperatura e pressão de operação estão nas vizinhanças do ponto crítico.
\end{abstract}

\section{INTRODUÇÃO}

Uma equação de estado é definida como uma relação entre as propriedades termodinâmicas de um fluido, sendo representada por uma relação matemática entre as funções de estado de um dado sistema (PIERRE, 1998). Através da modelagem termodinâmica pode-se representar o comportamento de propriedades como entalpia $(\mathrm{h})$, entropia (s), volume molar (v), calor específico isobárico (Cp), dentre outras propriedades correlatas, as quais são úteis para a descrição de vários processos (LEAL, 2012). Desse modo, as aplicações das equações de estado são de fundamental importância para o dimensionamento e a operações dos mais variados equipamentos na indústria química (LEAL, 2012), uma vez que é através do estudo e aplicação de tais equações que as informações para a determinação das propriedades termodinâmicas fundamentais são obtidas (SMITH et al., 2007).

Um estudo inicial sobre o comportamento das propriedades termodinâmicas foi realizado a partir da teoria cinética dos gases, resultando em uma primeira equação de estado conhecida como a equação do gás ideal. Tal equação relaciona pressão, temperatura e volume específico, e possui um bom desempenho quando aplicada a processos realizados a baixa pressão, como mostra a Eq. 1 abaixo:

$$
P V=n R T
$$


No entanto, o domínio de validade da Eq. 1 apresentada, não é válido quando o processo ocorre a pressões elevadas (FINLAYSON, 2006), quando se desvia da idealidade. Em tais condições, a equação do gás ideal não possui uma representação válida da realidade.

Com o objetivo de responder com maior exatidão ao comportamento dos gases reais, houve o desenvolvimento das equações de estado cúbicas, as quais são capazes de representar o comportamento dos processos mantendo um compromisso com entre a simplicidade e eficácia, sendo esta adequada a muitas aplicações (SMITH et al., 2007).

A equação de estado de van der Waals, Eq. 2, foi a primeira capaz de representar o equilíbrio de fases líquido-vapor (SANDLER, 1999).

$$
P=\frac{R \cdot T}{V-b}-\frac{a}{V^{2}}
$$

Diferente da equação ideal, a equação de van der Waals leva em consideração informações mais detalhadas sobre as moléculas dos sistemas. Onde o parâmetro $\boldsymbol{b}$ representa o volume de exclusão, ou seja, o volume ocupado pelas moléculas, e o $\boldsymbol{a}$ a força de interação existente entre as moléculas, como mostra a Tabela 1. Os resultados obtidos, com a aplicação desta equação, revelaram valores com uma maior precisão quando comparados com os obtidos experimentalmente, entretanto quando conduzido a pressões extremamente altas as aproximações não eram satisfatórias (FINLAYSON, 2006).

Desde então, algumas centenas de equações de estado cúbicas foram propostas. Dos muitos modelos desenvolvidos, desde 1873 com a equação de Van der Waals, apenas em 1949 foi formulado por Otto Redlich e Joseph Neng Shun Kwong um modelo capaz de descrever, com um maior grau de precisão, alguns sistemas. Tal equação de estado fícou conhecida como: equação de Redlich-Kwong, sendo considerada por muitos a melhor das equações de estado com dois parâmetros (Moran et al, 2013).

$$
P=\frac{R T}{V-b}+\frac{a / \sqrt{T}}{V(V)+b}
$$

A equação de Redlich-Kwong, em geral, é mais precisa do que a equação de Van der Waals, a temperaturas acima da temperatura crítica. Os parâmetros das equações estão descritos na Tabela 1 abaixo.

\begin{tabular}{|c|c|c|}
\hline EDE's & $\mathrm{a}$ & B \\
\hline \multirow[t]{2}{*}{ Van der Waals } & $27 \cdot R^{2} \cdot T c^{2}$ & R.TC \\
\hline & $64 . P C$ & $\overline{8 . P c}$ \\
\hline \multirow[t]{2}{*}{ Redlich-Kwong } & $0,42748 \cdot R^{2} \cdot T c^{2}$ & 0,08664.R.Tc \\
\hline & $P_{C}$ & $P_{c}$ \\
\hline \multirow{2}{*}{$\begin{array}{c}\text { Soave-Redlich- } \\
\text { Kwong }\end{array}$} & $0,42748 \cdot R^{2} \cdot T c^{2}$ & $0,08664 . R . T c$ \\
\hline & $P_{C}$ & $P c$ \\
\hline \multirow[t]{2}{*}{ Peng-Robinson } & $0,45724 \cdot R^{2} \cdot T c^{2}$ & 0,07780.R.TC \\
\hline & $P c$ & $P_{c}$ \\
\hline
\end{tabular}

Tabela 1-Parâmetros das Equações de Estado 
A equação de Redlich-Kwong foi concebida com o objetivo de prever as propriedades de moléculas pequenas, não-polares, em fase vapor, no entanto sendo ainda alvo de várias tentativas de revisões e modificações. Tais procedimentos objetivam o aumento da precisão em termos da previsão de propriedades em fase gasosa de um número maior de compostos, bem como na melhoria de condições que simulam a temperaturas mais baixas, incluindo equilíbrio líquido-vapor. Pode-se citar como exemplo, em 1975, onde foi publicada a adição de um terceiro parâmetro, a fim de melhorar o comportamento do modelo de ambas as moléculas de cadeia longa, bem como as moléculas mais polares, na qual consistia em tornar um parâmetro dependente da temperatura (REDLICH, 1949).

Contudo, as modificações mais substanciais precisavam ser feitas, e a mais bem sucedida foi a de Soave, proposta em 1972. Tal modificação envolveu a substituição do $T^{1 / 2}$ de energia encontrada no denominador do termo de interação molecular da equação original por uma expressão dependente da temperatura, como pode ser observado abaixo:

$\alpha(\mathrm{T})=\left[1+m \cdot\left(\sqrt{\frac{T}{T c}}\right)\right]^{2}$

$m=0,480+1,574 . \omega-0,176 \cdot \omega^{2}$

Todavia, os novos termos propostos, na então denominada equação de Soave RedlichKwong, são puramente empíricos e não apresenta bons resultados para misturas contendo fluidos polares e/ou com ponte de hidrogênio junto com gases comuns e hidrocarbonetos (LACERDA et al., 2000).

Em seguida, Peng e Robinson, em 1976, propuseram uma equação que apresentou um melhor desempenho, sobretudo nas vizinhanças do ponto crítico, e, também, para cálculos de densidade de líquido. Dessa forma, a equação de Peng-Robinson, Eq. 6 abaixo, pode ser aplicada em cálculos de propriedades termodinâmicas de fluidos puros e misturas (CARMO, 2009). Além disso, está entre as equações de estado de uso mais disseminado na indústria para simulação e modelagem termodinâmica de processos, devido a sua eficiência, simplicidade e facilidade de resolução (LEAL, 2012).

$P=\frac{R T}{V-b}+\frac{a \alpha(T)}{V^{2}+2 b V-b^{2}}$

Onde, os parâmetros da equação encontram-se na Tabela 1.

Diante do exposto, pergunta-se: quantas propriedades termodinâmicas são necessárias para caracterizar um sistema e como relaciona-las? A resposta está associada a precisão requerida pela definição do sistema e baseada nos objetivos do uso das equações de estado. Normalmente são definidas quatro propriedades, volume, temperatura, pressão e quantidade de massa, as quais podem ser relacionadas. Ao relaciona-se três dessas propriedades por uma equação de estado, a quarta sairá por inferência, diminuindo assim uma variável do processo e aumentando o grau de liberdade do sistema. Neste trabalho são definidas a pressão e a temperatura de operação com a finalidade de se obter o volume molar do sistema sob consideração. 


\section{METODOLOGIA}

Para a análise do comportamento das equações de estado, o processo de produção do metanol será analisado. Do ponto de vista industrial, o produto da reação é obtido a partir da reação de Monóxido de Carbono $(\mathrm{CO})$ com Hidrogênio $(\mathrm{H})$, conforme a reação abaixo:

$$
\mathrm{CO}+2 \mathrm{H}_{2} \rightarrow \mathrm{CH}_{4} \mathrm{O}
$$

Sendo assim, o processo de produção foi desenvolvido na plataforma de simulação Aspen Plus ${ }^{\circledR}$, e o flowsheet, como mostra a Figura 1, é composto por um reator do tipo RStic, o qual opera a 709,275 bar.

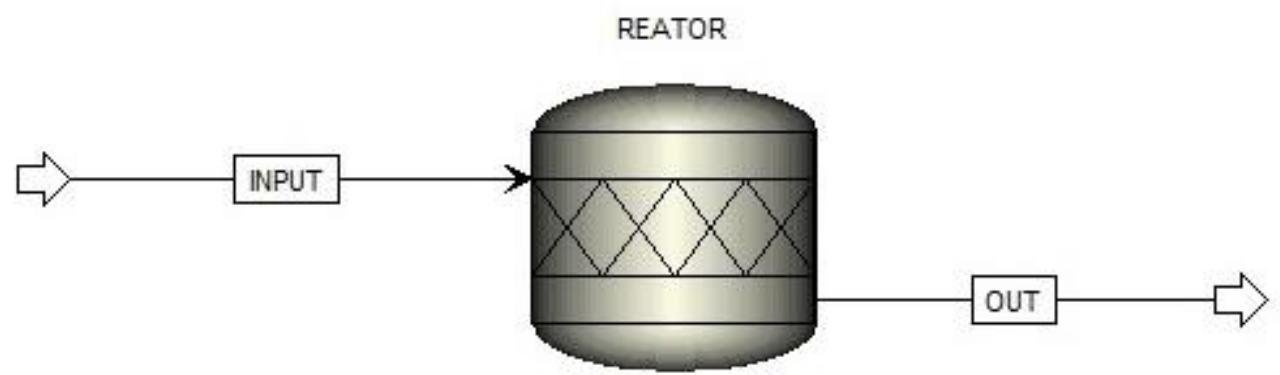

Figura 1 - Flowsheet do processo no Aspen Plus ${ }^{\circledR}$

Cada uma das equações de estado mencionadas na Tabela 1 foi utilizada, a saber: Redlich-Kwong, Soave-Redlich-Kwong, Peng-Robinson, Van der Waals e a Lei dos Gases Ideais, e na Tabela 2, encontram-se as informações utilizadas para a corrente de alimentação:

\begin{tabular}{cc}
\multicolumn{2}{c}{ Tabela $2-$ Dados utilizados } \\
\hline Fluxo de $\mathrm{CO}(\mathrm{kmol} / \mathrm{hr})$ & 100 \\
\hline Fluxo de $\mathrm{H}_{2}(\mathrm{kmol} / \mathrm{hr})$ & 200 \\
\hline Fluxo de metanol $(\mathrm{kmol} / \mathrm{hr})$ & 100 \\
\hline Pressão $(\mathrm{atm})$ & 100 \\
\hline Temperatura $\left({ }^{\circ} \mathrm{C}\right)$ & 300 \\
\hline
\end{tabular}

\section{RESULTADOS E DISCUSSÃO}

Na Tabela 2 são representadas as condições de alimentação do processo para produção de metanol, além dos resultados obtidos da plataforma Aspen Plus para as equações de estados consideradas e apresentadas na Tabela 3. 
Tabela 3 - Condições de processo de metanol no flowsheet para as diferentes equações de estado.

\begin{tabular}{|c|c|c|c|c|c|c|c|c|c|c|}
\hline & \multicolumn{2}{|c|}{$\begin{array}{l}\text { Lei dos Gases } \\
\text { Ideais }\end{array}$} & \multicolumn{2}{|c|}{ Van der Waals } & \multicolumn{2}{|c|}{ Redlich-Kwong } & \multicolumn{2}{|c|}{$\begin{array}{l}\text { Soave-Redlich- } \\
\text { Kwong }\end{array}$} & \multicolumn{2}{|c|}{ Peng-Robinson } \\
\hline & Input & Out & Input & Out & Input & Out & Input & Out & Input & Out \\
\hline Substream: MIXED & & & & & & & & & & \\
\hline $\begin{array}{c}\text { Phase } \\
\text { Component Mole Flow }\end{array}$ & Vapor & Vapor & Vapor & Vapor & Vapor & Vapor & Vapor & Vapor & Vapor & Vapor \\
\hline CARBO-01 (Kmol/hr) & 100,0 & 20,0 & 100,0 & 20,0 & 100,0 & 20,0 & 100,0 & 20,0 & 100,0 & 20,0 \\
\hline HYDRO-01 (Kmol/hr) & 200,0 & 40,0 & 200,0 & 40,0 & 200,0 & 40,0 & 200,0 & 40,0 & 200,0 & 40,0 \\
\hline METHA-01 (Kmol/hr) & 100,0 & 180,0 & 100,0 & 180,0 & 100,0 & 180,0 & 100,0 & 180,0 & 100,0 & 180,0 \\
\hline Mole Flow (Kmol/hr) & 400,0 & 240,0 & 400,0 & 240,0 & 400,0 & 240,0 & 400,0 & 240,0 & 400,0 & 240,0 \\
\hline Mass Flow (Kg/hr) & $\begin{array}{c}6408,4 \\
32\end{array}$ & $\begin{array}{c}6408,4 \\
32\end{array}$ & $\begin{array}{c}6408,4 \\
32\end{array}$ & $\begin{array}{c}6408,4 \\
32\end{array}$ & $\begin{array}{c}6408,4 \\
32\end{array}$ & $\begin{array}{c}6408,4 \\
32\end{array}$ & $\begin{array}{c}6408,4 \\
32\end{array}$ & $\begin{array}{c}6408,4 \\
32\end{array}$ & $\begin{array}{c}6408,4 \\
32\end{array}$ & $\begin{array}{c}6408,4 \\
32\end{array}$ \\
\hline Volume Flow (L/min) & $\begin{array}{c}3135,3 \\
62\end{array}$ & $\begin{array}{c}449,19 \\
83\end{array}$ & $\begin{array}{c}3172,0 \\
74\end{array}$ & $\begin{array}{c}505,96 \\
47\end{array}$ & $\begin{array}{c}3225,5 \\
21\end{array}$ & $\begin{array}{c}578,23 \\
79\end{array}$ & $\begin{array}{c}3240,7 \\
25\end{array}$ & $\begin{array}{c}576,26 \\
82\end{array}$ & $\begin{array}{c}3195,4 \\
33\end{array}$ & $\begin{array}{c}549,89 \\
10\end{array}$ \\
\hline Temperature (K) & 573,15 & 958,0 & 573,15 & 958,0 & 573,15 & 958,0 & 573,15 & 958,0 & 573,15 & 958,0 \\
\hline Pressure (Atm) & 100,0 & 700,0 & 100,0 & 700,0 & 100,0 & 700,0 & 100,0 & 700,0 & 100,0 & 700,0 \\
\hline Vapor Fraction & 1,0 & 1,0 & 1,0 & 1,0 & 1,0 & 1,0 & 1,0 & 1,0 & 1,0 & 1,0 \\
\hline $\begin{array}{l}\text { Molar Enthalpy } \\
\qquad(\mathrm{Cal} / \mathrm{mol})\end{array}$ & $\begin{array}{c}- \\
16257 \\
90\end{array}$ & $\begin{array}{c}- \\
28998, \\
53\end{array}$ & $\begin{array}{c}- \\
16331 \\
13\end{array}$ & $\begin{array}{c}- \\
29829 \\
89\end{array}$ & $\begin{array}{c}- \\
16331, \\
12\end{array}$ & $\begin{array}{c}- \\
29360 \\
55\end{array}$ & $\begin{array}{c}- \\
16314, \\
60\end{array}$ & $\begin{array}{c}- \\
29402 \\
53\end{array}$ & $\begin{array}{c}- \\
16345, \\
16\end{array}$ & $\begin{array}{c}- \\
29633, \\
33\end{array}$ \\
\hline
\end{tabular}


Pode-se observar, através dos resultados, que houve uma pequena variação no volume molar para cada equação calculada. Os valores obtidos dependem das características do sistema e da equação de estado utilizada. Como o sistema em questão está operando à temperatura e pressão próximas dos pontos críticos, pela literatura, a equação que melhor representa o sistema sob tais condições é a de Peng-Robinson, visto que leva em consideração parâmetros relacionados as propriedades críticas da substância em questão.

Para uma melhor visualização, a Tabela 4 abaixo fornece os valores dos erros percentuais das equações de estados com relação à equação de Peng-Robinson que foi utilizada como padrão de referência.

Tabela 4 - Erros percentuais das equações de estado com base no valore da equação de PengRobinson para o Metanol na corrente de saída (out).

\begin{tabular}{cc}
\hline Lei dos Gases Ideais & $18,31 \%$ \\
\hline Van der Waals & $7,99 \%$ \\
\hline Redlich-Kwong & $5,15 \%$ \\
\hline Soave-Redlich-Kwong & $4,80 \%$ \\
\hline
\end{tabular}

\section{CONCLUSÃO}

A comparação do desempenho de equações de estado é uma das etapas importantes para a definição da mais adequada para a determinação de propriedades termodinâmicas, principalmente em situações onde não tem-se apenas substâncias puras. A predição de tais propriedades, no caso do trabalho desenvolvido o volume específico, é de suma importância para a indústria de processos químicos, pois estes cálculos são imprescindíveis para avaliação e desenvolvimento de projetos, processos e para determinação de outras propriedades termodinâmicas correlatas no sistema. Dentro deste escopo, as equações de estado PengRobinson, Soave-Redlich-Kwong, Redlich-Kwong, Van der Waals e a Lei Dos Gases Ideais foram testadas e seus respectivos volumes foram calculados. Dado que cada equação de estado possui um domínio de validade, observa -se variações no volume molar calculado.

As características da equação, bem como os resultados obtidos indicam que a equação de estado Peng-Robinson representa o processo analisado, com um maior grau de precisão o comportamento do volume molar nas condições de operação apresentadas (fases gasosa e condições supercríticas), levando em consideração que dentre as equações de estado, a equação de Peng-Robinson é, segundo a literatura, a equação que melhor prever as propriedades termodinamicas nessas condições. Logo, pode-se concluir que, devido sua ampla faixa de aplicabilidade, esta equação de estado é a mais indicada para o estudo e determinação de propriedades termodinâmicas, tanto pela sua simplicidade funcional como pela representação quase fiel à realidade nessas condições. 


\section{REFERÊNCIAS BIBLIOGRÁFICAS}

1. CARMO, F. R., Desenvolvimento de ambiente computacional para cálculos termodinâmicos de substâncias puras e misturas e modelagem de equilíbrio líquidovapor a pressões baixas e moderadas utilizando equações de estado cúbicas. Monografia-Universidade Federal do Ceará, Fortaleza, 2009.

2. FINLAYSON, B. A., Introduction to Chemical Engineering Computing. Wiley Interscience, New Jersey, 2006

3. LACERDA, R., EMBIRUÇU, M., COSTA, G., Equação de estado. Universidade Federal da Bahia, Bahia, 2000.

4. LEAL, D. S., Avaliação de equações de estado no cálculo de propriedades do dióxido de carbono úteis ao estudo do seu escoamento. Tese de Mestrado, Universidade Federal da Bahia, Salvador, 2012.

5. MURDOCK, J. W., Fundamental fluid mechanics for the practicing engineer. CRC Press, p. 25-27, 1993.

6. PIERRE, P., A to Z of Thermodynamics. Oxford University Press, 1998.

REDLICH, O., KWONG, J. N. S., On thermodynamics of solution. V: An equation of state. Fugacities of gaseous solutions. Chem. Rev., v.44, p.233, 1949.

7. SMITH, J. M., VAN NESS, H. C., ABBOTT, M. M., Tradução QUEIROZ, E. M., Introdução á Termodinâmica da Engenharia Química. 7ed, Rio de Janeiro, LTC, 2007.

8. SANDLER, S. I., Chemical and Engineering Thermodynamics, $3^{\text {a }}$ Edição, John Wiley \& Sons, 1999.

9. MORAN, MICHAEL J.; SHAPIRO, HOWARD N.; BOETTNER, DAISIE D.; BAILEY, MARGARET B. Principios de Termodinamica para Engenharia. $7^{\mathrm{a}}$ ed., Ed. LTC, 2013. 